\title{
Nasal ventilation in pregnancy: treatment of nocturnal hypoventilation in a patient with kyphoscoliosis
}

\author{
L.J. Restrick, B.R. Clapp, C. Mikelsons, J.A. Wedzicha
}

\begin{abstract}
Nasal ventilation in pregnancy: treatment of nocturnal hypoventilation in a patient with kyphoscoliosis. L.J. Restrick, B.R. Clapp, C. Mikelsons, J.A. Wedzicha. @ERS Journals Ltd 1997.

ABSTRACT: The management of a young woman with congenital kyphoscoliosis, who developed symptomatic nocturnal hypoventilation during the third trimester of pregnancy, is described.

Nasal intermittent positive pressure ventilation (NIPPV) was safely and effectively used to correct nocturnal hypoxaemia and hypercapnia from the 30th-36th week of gestation, when a healthy boy was delivered by Caesarean section. Following delivery, the mother no longer required NIPPV and returned to her prepregnancy level of activity.

Eur Respir J 1997; 10: 2657-2658.
\end{abstract}

\author{
Academic Dept of Respiratory Medicine, \\ London Chest Hospital, London, UK. \\ Correspondence: J.A. Wedzicha \\ Academic Dept of Respiratory Medicine \\ London Chest Hospital \\ Bonner Road \\ London E2 9JX \\ UK
}

Keywords: Kyphoscoliosis, nasal ventilation, nocturnal hypoventilation, pregnancy

Received: April 301997

Accepted after revision July 301997
Patients with severe kyphoscoliosis commonly develop nocturnal hypoventilation which may progress to respiratory failure and cor pulmonale [1,2]. Nasal intermittent positive pressure ventilation (NIPPV) is an effective method of providing nocturnal ventilatory support in this group of patients $[3,4]$. Pregnancy is not uncommon in patients with kyphoscoliosis, and is usually well tolerated [5, 6]. However, pregnancy may precipitate respiratory failure in patients with severe kyphoscoliosis [7]. Whilst negative pressure ventilation has been used successfully to treat respiratory failure complicating pregnancy in these patients [7], the effect of NIPPV on nocturnal hypoventilation developing in pregnancy has not been described.

\section{Case report}

A 32 year old woman with congenital kyphoscoliosis was referred at 26 weeks of pregnancy due to increasing shortness of breath. She had a mid-thoracic kyphoscoliosis, with an initial Cobb angle of more than $90^{\circ}$. When the patient was 12 yrs of age, a Harrington rod was positioned and, at 17 yrs of age, bilateral costoplasty was performed. Prepregnancy, she worked fulltime as a teacher and was able to walk up two flights of stairs without stopping. Two years previously, she had a spontaneous abortion at 13 weeks of pregnancy. She had mild asthma, was a nonsmoker, did not drink alcohol and was receiving no medication.

On examination, the patient was short (height $142 \mathrm{~cm}$, nonpregnant weight $44 \mathrm{~kg}$ ) with a marked thoracic kyphoscoliosis $\left(\mathrm{Cobb}\right.$ angle $60^{\circ}$ ). She had a regular pulse of 82 beats $\mathrm{min}^{-1}$, her blood pressure when lying was $110 / 70 \mathrm{mmHg}$, jugular venous pressure was elevated at $3 \mathrm{~cm}$, and there was a loud pulmonary component of the second heart sound. Expansion was reduced bilaterally but breath sounds were vesicular and she had no peripheral oedema. She had no hepatomegaly and a gravid uterus compatible with 26 weeks gestation. She had a forced expiratory volume in one second (FEV1) of $0.77 \mathrm{~L}$ (34\% of predicted value) and a forced vital capacity (FVC) of 0.87 L (33\% pred). Daytime arterial oxygen tension $\left(\mathrm{Pa}_{\mathrm{a}} \mathrm{O}_{2}\right)$ was $10.2 \mathrm{kPa}$ and arterial carbon dioxide tension $\left(\mathrm{Pa}_{\mathrm{a}} \mathrm{CO}_{2}\right)$ was $5.8 \mathrm{kPa}$. An electrocardiogram (ECG) showed right axis deviation and right ventricular hypertrophy.

An overnight study was performed at 28 weeks, using the Edentrace multichannel recording system (Nellcor Puritan Bennett, Minnesota, USA). This showed a mean arterial oxygen saturation $\left(\mathrm{Sa}_{\mathrm{a}} \mathrm{O}_{2}\right)$ of $90 \%$, including three episodes of desaturation: one of 12 min with a minimum $\mathrm{Sa}_{\mathrm{a}} \mathrm{O}_{2}$ of $79 \%$; one of 18 min with a minimum $\mathrm{Sa}_{\mathrm{a}} \mathrm{O}_{2}$ of $72 \%$; and one of $22 \mathrm{~min}$ with a minimum $\mathrm{Sa}_{\mathrm{a}} \mathrm{O}_{2}$ of $70 \%$. The reductions in oronasal airflow and chest wall movement were consistent with hypoventilation, although transcutaneous partial pressure of carbon dioxide $\left(P \mathrm{tc}, \mathrm{CO}_{2}\right)$ was not recorded. By 30 weeks, the patient was more symptomatic with "chest tightness" when lying down, and was waking unrefreshed. Daytime $P \mathrm{a}, \mathrm{O}_{2}$ was $10.2 \mathrm{kPa}$ and $P \mathrm{a}, \mathrm{CO}_{2}$ had increased to 6.1 $\mathrm{kPa}$. She was, therefore, established on NIPPV using the bilevel positive airway pressure (BiPAP) (Respironics Inc., Murrysville, PA, USA) nasal ventilator in the spontaneous (S) mode, initially as an in-patient. Her symptoms improved dramatically and a repeat sleep study showed a mean $\mathrm{Sa}_{\mathrm{a}} \mathrm{O}_{2}$ of $92 \%$, minimum $\mathrm{Sa}_{\mathrm{a}} \mathrm{O}_{2}$ of $90 \%$ and normal oronasal airflow and chest wall movement.

The patient tolerated NIPPV well, with an inspiratory positive airway pressure (IPAP) of $16 \mathrm{cmH}_{2} \mathrm{O}$ and expiratory positive airway pressure (EPAP) of $4 \mathrm{cmH}_{2} \mathrm{O}$. However, by 35 weeks she was again feeling tired. Daytime $P$ a, $\mathrm{O}_{2}$ was now $9.6 \mathrm{kPa}$ and $P \mathrm{a}_{1} \mathrm{CO}_{2}$ was $6.4 \mathrm{kPa}$. As she was not comfortable with a further increase in 
IPAP and her daytime $P \mathrm{a}, \mathrm{CO}_{2}$ was less than $7 \mathrm{kPa}$, nocturnal oxygen at $1 \mathrm{~L} \cdot \mathrm{min}^{-1}$ was added via the BiPAP ventilator, with marked symptomatic benefit. A repeat sleep study showed a mean $\mathrm{Sa}_{\mathrm{a}} \mathrm{O}_{2}$ of $95 \%$, minimum $\mathrm{Sa}_{\mathrm{a}, \mathrm{O}_{2}}$ of $91 \%$, and with acceptable hypercapnia (mean $P \mathrm{tc}, \mathrm{CO}_{2} 7.7 \mathrm{kPa}$ ), measured by the TINA $P \mathrm{tc}, \mathrm{CO}_{2}$ monitoring system (Radiometer, Copenhagen, Denmark).

At 36 weeks, the patient was admitted for an elective Caesarean section under general anaesthetic, and delivered a healthy baby boy (3.15 kg, Apgar score 10)). NIPPV was continued for 1 week. Four months postpartum, she was back to her prepregnancy level of activity, with an FEV1 of $0.80 \mathrm{~L}$ (35\% pred), FVC of 1.00 $\mathrm{L}$ (38\% pred), daytime $\mathrm{Pa}, \mathrm{O}_{2}$ of $11.9 \mathrm{kPa}$ and $P \mathrm{a}, \mathrm{CO}_{2}$ of $5.7 \mathrm{kPa}$. A sleep study breathing air showed nocturnal hypoventilation, with mean $\mathrm{Sa}_{2} \mathrm{O}_{2}$ of $93 \%$, a minimum $\mathrm{Sa}_{\mathrm{a}} \mathrm{O}_{2}$ of $89 \%$, and mean $\mathrm{Ptc}_{\mathrm{tc}} \mathrm{CO}_{2} 9.7 \mathrm{kPa}$. As the patient has reduced lung volumes and persistent nocturnal hypoventilation postpartum, with significant hypercapnia, she is likely to require home nocturnal ventilatory support in the future.

\section{Discussion}

Patients with severe kyphoscoliosis often present with acute respiratory failure against the background of a long-standing deformity that has previously been associated with relatively few symptoms. While prepregnancy baseline data were not available in this case, nocturnal hypoventilation was documented at follow-up postpartum. It is likely that the physiological changes of the third trimester of pregnancy resulted in worsening of underlying nocturnal hypoventilation due to kyphoscoliosis, which then improved postpartum.

Using polysomnography, which is the recognized gold standard for the investigation of sleep-related breathing disorders, nocturnal hypoventilation has been shown to occur, in patients with kyphoscoliosis particularly during rapid eye movement sleep $[1,2]$. It is most likely to be due to reduced inspiratory muscle activity of the accessory muscles and diaphragm, which may already be at a mechanical disadvantage as a result of the chest wall deformity $[1,2,4]$. Worsening nocturnal hypoventilation in the third trimester of pregnancy, which improves postpartum, is a likely consequence of the upwards displacement of the diaphragm by the foetus. Polysomnography does not usually alter the management of patients demonstrating typical patterns of sleep disturbance, and its use is usually reserved for those patients where there is diagnostic uncertainty despite limited sleep studies or a poor response to treatment.

The dramatic response to NIPPV in this patient is typical of that of nonpregnant patients with congenital kyphoscoliosis treated with NIPPV [3, 4]. In contrast, overnight oxygen therapy may result in severe hypercapnia, and, therefore, has to be used very cautiously in patients with kyphoscoliosis [2]. With the respiratory support provided by NIPPV, it was possible to use supplemental oxygen in this patient without causing unacceptable hypercapnia, so maximizing oxygen delivery to the foetus. NIPPV was a safe, well-tolerated, and effective treatment of nocturnal hypoventilation precipitated by pregnancy in this patient with kyphoscoliosis. Pregnancy is not contra-indicated in the majority of patients with kyphoscoliosis [5-7]. However, in patients who develop symptomatic nocturnal hypoventilation or respiratory failure during pregnancy, nocturnal intermittent positive pressure ventilation should be considered.

\section{References}

1. Mezon BL, West P, Israels J, Kryger M. Sleep breathing abnormalities in kyphoscoliosis. Am Rev Respir Dis 1980; 122: 617-621.

2. Sawicka EH, Branthwaite MA. Respiration during sleep in kyphoscoliosis. Thorax 1987; 42: 801-808.

3. Carroll N, Branthwaite MA. Control of nocturnal hypoventilation by nasal intermittent positive pressure ventilation. Thorax 1988; 43: 349-353.

4. Ellis ER, Grunstein RR, Chan S, Bye PTP, Sullivan CE. Noninvasive ventilatory support during sleep improves respiratory failure in kyphoscoliosis. Chest 1988; 94 : 811-815.

5. Manning CW, Prime FJ, Zorab PA. Pregnancy and scoliosis. Lancet 1967; ii: 792-795.

6. Siegler D, Zorab PA. Pregnancy in thoracic scoliosis. Br J Dis Chest 1981; 75: 367-370.

7. Sawicka EH, Spencer GT, Branthwaite MA. Management of respiratory failure complicating pregnancy in severe kyphoscoliosis: a new use for an old technique. $\mathrm{Br} J$ Dis Chest 1986; 80: 191-196. 